\title{
The Effect of Coffee Consumption Behavior on Increased Blood Pressure
}

\author{
Karina Kaptelova ${ }^{1}$, Elena Pysanka ${ }^{1}$ \\ ${ }^{1}$ Medical faculty, Uzhhorod National University, Ukraine
}

\begin{abstract}
The purpose of this research is to establish whether or not coffee has an adverse impact on blood pressure. Analytic observation was used in this kind of study, which was carried out utilizing a cross-sectional methodology and analytic observation. The findings were similar with individuals who had never experienced hypertension. Also, the proportion of healthy individuals was greater. A research shows that coffee consumption raises blood pressure. The research comprised 42 people who drank coffee three times or more per day and had high blood pressure ( 80.7 of the time) The chi-square test revealed that coffee consumption raises blood pressure by $\mathrm{p}=0.000$.
\end{abstract}

Keywords: Coffee, Blood Pressure, Caffeine

Received : September 7, 2021

Received in Revised: September 25, 2021

Accepted: October 2, 2021

\section{Introduction}

The effort made by the blood to flow through the blood vessels is referred to as blood pressure. Arteries are blood arteries that transport blood from the heart to the rest of the body. They are also known as capillaries. Blood pressure is measured in two ways: systolic and diastolic, with normal values less than or equal to $120 / 80 \mathrm{mmHg}$ for both measurements (millimetres of mercury). According to WHO statistics, about 972 million people worldwide, or 26.4 percent of the world's population, suffer with hypertension, a number that is expected to rise to 29.2 percent by 2025 , based on current trends. Of the 972 million individuals who have hypertension, 333 million live in industrialized countries, with the remainder 639 living in underdeveloped nations. Because hypertension is one of the leading causes of death and morbidity in Indonesia, the treatment of this illness is a highly frequent intervention that is carried out at all levels of health care.

Coffee is a beverage that has been enjoyed since ancient times and is now one of the most popular beverages consumed throughout the globe (Weinberg \& Bealer, 2004). Because coffee is extensively used in society, even the smallest effect of coffee on blood pressure will have an influence on public health because of this. In addition to affecting the growth of the corporate world, coffee's popularity has also had an impact on the development of the culinary industry (Mussatto et al., 2011). Robusta coffee accounts for the majority of coffee produced and sold; this kind of coffee has a caffeine level that is 2-3 percent greater than that of other coffees, which is 1-1.3 percent. Coffee that enters the body is transported throughout the body via the bloodstream, which travels from the gastrointestinal tract in about 5-15 minutes after entering the body. It takes 45-60 minutes for caffeine absorption to reach a peak in the circulation once it has reached 99 percent absorption levels in the digestive system. Caffeine is very efficient at functioning in the body, and as a result, it has a wide range of impacts on the body (Grgic et al 2019; Grgic et al 2018; Goldstein et al., 2010).

The amount of caffeine in coffee varies based on the kind of coffee, the origin of the coffee, the environment in which the coffee is grown, and the method used in the coffee processing

Copyright @ 2021, International Journal Papier Advance and Scientific Review, Under the license CC BY-SA 4.0

DOI: $\underline{\text { https://doi.org/10.47667/ijpasr.v2i2.111 }}$ 
process. Those who have a daily habit of consuming 1-2 cups of coffee per day have a 4.12 times greater chance of developing hypertension than people who do not have a daily practice of drinking coffee. Because of the polyphenols, potassium, and caffeine included in coffee, it has the potential to raise or lower blood pressure.

Potassium and polyphenols both help to decrease blood pressure. Polyphenols are antiatherosclerotic and have been shown to enhance vascular function. Sodium and water are excreted more readily by the body when potassium is present. Potassium lowers both systolic and diastolic blood pressure by blocking the release of renin. Plasma volume, cardiac output, and peripheral pressure all drop as a result, and blood pressure falls as a result of these changes. Caffeine has an antagonistic action on adenosine receptors that is competitive in nature. Adenosine is a neuromodulator that has the ability to modulate a wide range of activities in the central nervous system (Choi et al., 2019; Choudhury et al., 2019; Wei et al., 2011). This has an effect on vasoconstriction and raises total peripheral resistance, which will cause blood pressure to rise as a result of the increased resistance.

According to the epidemiology, it was discovered that coffee intake was inconsistent with hypertension, with some studies showing a positive connection and others showing a lack of a relationship. This is due to the presence of polyphenols and potassium in coffee, both of which may lower blood pressure and act as antioxidants, thus reducing the amount of harmful free radicals in the body.

Furthermore, the mean pulse pressure must be maintained in order to maintain smooth systemic blood flow, and the optimum blood pressure limit must be maintained in order to provide sufficient perfusion of oxygen and nutrients from the capillaries to the tissues. Average pulse pressure must be maintained at an optimal level because it is not only important for maintaining good perfusion, but it is also important for preventing the heart from working harder and causing damage to blood vessels. If the average pulse pressure is too high, this can result in heart failure.

High blood pressure is caused by a hormonal process, namely the production of angiotensin II from angiotensin I by the enzyme Angiotensin Converting Enzyme (ACE). ACE is a hormone that has a significant physiological function in the regulation of blood pressure. Angiotensinogen is a substance found in the blood that is generated by the liver. Aside from that, the kidneys' hormone renin will be transformed into angiotensinogen I, which will be released into the bloodstream. Angiotensin I is transformed into angiotensin II in the lungs by angiotensin converting enzyme (ACE) (Kaparianos \& Argyropoulou, 2011). Angiotensin II is the hormone that plays a critical part in the elevation of blood pressure via two distinct mechanisms.

Caffeine has a half-life of 3 to 7 hours, and this may have an adverse effect on a person's ability to sleep well. Individuals suffering from cardiac disease should avoid caffeine use. The use of caffeine by patients with renal illness should be limited since the diuretic nature of caffeine may exacerbate the patient's condition. Despite the fact that no link has been shown between caffeine use and congenital anomalies in research performed to far, pregnant women are advised to avoid caffeine consumption. Because of the acidic nature of caffeine, patients suffering from stomach ulcers and other gastrointestinal disorders should exercise caution while taking the stimulant. Several excellent articles have been published on the impact of the autonomic nervous system on coffee consumption. Caffeine exerts its effect by stimulating the nonandrogenic nerves in the surrounding area, which in turn stimulates the sympathetic 
nervous system (Omondi, 2011). As a result, after drinking coffee or caffeine, the heart rate rises somewhat higher than normal. The majority of researchers come to the same conclusion.

Although opinions on the impact of coffee on blood pressure are still divided, one thing is certain: Caffeinated coffee produced a fast but statistically significant rise in both systolic and diastolic blood pressure when he investigated the acute effects of both caffeinated and decaffeinated coffee on hemodynamic (Buscemi et al., 2010; Papamichael et al., 2005). Effect of coffee on endothelial function in healthy subjects: the role of caffeine. Clinical Science, 109(1), 55-60.)

\section{Methods}

The technique of analytical observation was used to perform this kind of research project. It was decided to use a cross-sectional method for this investigation. In order to characterize the properties of the independent and dependent variables, a univariate analysis is performed. Every piece of information collected by means of the questionnaire is processed and shown in the form of a frequency distribution table. Chi-square test analysis was performed to see if there was a potential connection between the independent and dependent variables in this bivariate study of data collection. It will be possible to get a $\mathrm{p}$ value via the application of the Kolmogorof Smirnov statistical test, with a significance level of 0.05 being utilized in this study. In research, a study is considered significant if it yields a p value of less than 0.05 , which indicates that Ho is rejected and $\mathrm{Ha}$ is accepted; a study is considered meaningless if it yields a $\mathrm{p}$ value greater than 0.05 , which indicates that $\mathrm{Ho}$ is accepted and $\mathrm{Ha}$ is rejected.

\section{Results and Discussion}

Men between the ages of 20 and 40 are required as research participants or samples. According to the findings of the sample size calculation, a total of 44 individuals are required for the investigation. Sixty individuals participated in this research as part of the sample. The kind of coffee, the amount of coffee, and the frequency of coffee consumption will all be calculated based on the completed sample. A change in blood pressure, a rise in blood pressure, or a reduction in blood pressure are all decided based on the findings of the measures of blood pressure taken after that.

\section{Univariate Analysis}

The univariate analysis stage is the initial step of data analysis. In order to obtain a description of the frequency distribution of each of the variables studied, a univariate analysis was performed. This included the characteristics of the respondents, such as the distribution of respondents based on the group history of high blood pressure, hypertension drug consumption, family history of hypertension, history of other diseases, family history of disease, and smoking, as well as the characteristics of the respondents.

After conducting an analysis, it was discovered that the distribution of samples based on a history of hypertension and history of not hypertension was 11.7 percent ( 7 samples) in respondents who had a history of hypertension and 88.3 percent ( 88 samples) in respondents who had a history of not hypertension (based on the results of the analysis conducted) (53 samples). According to the distribution of samples based on those who took hypertension medications and those who did not take hypertension medications, that is, the percentage of those who took hypertension medications was 5.0 percent $(3$ samples) and the percentage of those who did not take hypertension medications was 95.0 percent (57 samples). According to the distribution of samples based on family history who have a history of hypertension versus 
samples who do not have a history of hypertension, the percentage of samples with a family history of hypertension is 5.0 percent ( 3 samples), and the percentage of samples with a family history of not having hypertension is 95.0 percent (nine samples) (57 samples).

As determined by the distribution of samples based on those who have and do not have a history of other illnesses, the percentage of those who have a history of other diseases is 5.0 percent ( 3 samples), while the percentage of those who do not have a history of other diseases is 95.0 percent (57 samples). In accordance with the distribution of samples according to family history of illness, the proportion of samples with a family history of disease is 1.7 percent, according to the data ( 1 sample). And the proportion of samples that do not include any other illnesses is 98.3 percent, according to the data (59 samples). According to the distribution of samples based on their smoking history, the proportion of samples with a history of smoking was 86.7 percent (51 samples), and the number of samples without a smoking history was 13.3 percent (13 samples) (9 samples).

\section{Bivariate Analysis}

Bivariate analysis was performed in order to determine the connection between the independent variables (kind of coffee, quantity of coffee, and frequency of coffee consumption) and the dependent variable (frequency of coffee consumption) (blood pressure). The chi-square test was used to conduct this investigation.

Table 1. Effect of Type of Coffee on Increased Blood Pressure

\begin{tabular}{|c|c|c|c|c|c|c|}
\hline & $\mathbf{N}$ & $\mathbf{\%}$ & $\mathbf{N}$ & $\mathbf{\%}$ & $\mathbf{n}$ & $\mathbf{\%}$ \\
\hline Instant Coffee & 1 & 6,25 & 15 & 93,7 & 16 & 100,0 \\
\hline Coffee & 43 & 97,7 & 1 & 2,27 & 44 & 100,0 \\
\hline Black Coffee/Ground Coffee & & & & & 0.000 & \\
\hline Total & 44 & & 16 & & 60 & \\
\hline
\end{tabular}

The findings of an investigation on the relationship between coffee intake and elevated blood pressure are shown in the table above. On the basis of these findings, it seems that coffee intake has an impact on blood pressure elevation. There were total of 60 individuals who drank coffee, and there were total of 60 people who had their blood pressure monitored.

One person (62.5 percent) reported a rise in blood pressure after drinking instant coffee, according to the data above, and 43 individuals reported an increase in blood pressure after ingesting ground coffee, according to the data (97.7 percent). Meanwhile, the number of persons who did not notice a change in blood pressure after drinking instant coffee was just 15 individuals (93.7 percent). For the sample, there was just one individual who did not notice an elevation in blood pressure after eating ground coffee (2.27 percent).

Two individuals participated in the study and reported a rise in blood pressure after drinking $300 \mathrm{gr}$ or more of coffee with a lot of caffeine (11.1 percent). The number of individuals who did not notice changes in blood pressure after drinking $300 \mathrm{gr}$ of coffee was sixteen $(88.8$ percent). There were 42 individuals (100.0 percent) in the samples who did not experience an increase in blood pressure after eating coffee less than $300 \mathrm{~g}$, and there were 0 persons in the samples who did experience an increase in blood pressure after ingesting coffee more than 300 g. (0 percent).

42 individuals were included in the study because they consumed coffee more than or equal to three times a day and noticed an elevation in blood pressure (80.7 percent). The number of individuals who did not experience changes in blood pressure while drinking coffee more than

Copyright @ 2021, International Journal Papier Advance and Scientific Review, Under the license CC BY-SA 4.0

DOI: $\underline{\text { https://doi.org/10.47667/ijpasr.v2i2.111 }}$ 
or equal to three times a day was ten in total throughout the study (19.2 percent). Two individuals participated in the study and noticed a rise in blood pressure when they consumed coffee fewer than three times a day ( 25 percent). Six individuals participated in the study who did not notice changes in blood pressure when they had coffee fewer than three times a day $(7$ percent).

The findings of statistical testing utilizing the chi-square test for the impact of drinking coffee on raising blood pressure were $\mathrm{p}=0.000(\mathrm{p}=0.05)$ for the effect of drinking coffee on increasing blood pressure. Blood pressure increases in direct proportion to the quantity of coffee consumed, with $\mathrm{p}=0.000(\mathrm{p}=0.05)$. Because it is statistically possible to infer that coffee intake has an impact on raising blood pressure, hypothesis zero is rejected and hypothesis one is accepted.

\section{Effect Of Coffee Type on Increasing Blood Pressure}

Specifically, the findings of this research show an association between coffee intake and increased blood pressure. Additionally, the findings of this study indicate that there is a relationship between the kind of coffee consumed and increased blood pressure. The study technique used is observational analytic with a cross-sectional approach, with a total sample size of 60 individuals between the ages of 20 and 40 years being studied.

Table 2. Stages of Blood Pressure

\begin{tabular}{|c|c|c|c|}
\hline Blood Pressure Category & \multicolumn{2}{|c|}{ Systolic mm Hg (Upper\#) } & Diastolic mm Hg (Lower\#) \\
\hline Normal & Less than 120 & And & Less than 80 \\
\hline Elevated & $120-129$ & And & Less than 80 \\
\hline $\begin{array}{c}\text { High Blood Pressure } \\
\text { (Hypertension) Stage 1 }\end{array}$ & $130-139$ & Or & $80-89$ \\
\hline $\begin{array}{c}\text { High Blood Pressure } \\
\text { (Hypertension) Stage 2 }\end{array}$ & 140 or Higher & Or & 90 or higher \\
\hline $\begin{array}{c}\text { Hypertensive Crisis } \\
\text { (Require Emergency Care) }\end{array}$ & Higher than 180 & And/or & Higher than 120 \\
\hline
\end{tabular}

There are two kinds of coffee: instant coffee and brewed/black coffee. Instant coffee is the most common form. The impure coffee that the subject consumes is instant coffee, which is a combination of coffee, creamer, and sugar that is eaten in a single serving. Approximately 10 $\mathrm{mg}$ of calories, 2 grams of carbs, $500 \mathrm{mg}$ of sugar, $500 \mathrm{mg}$ of fat, and $5 \mathrm{mg}$ of salt are included in a tablespoon of creamer. Although the poor nutritional value of this creamer has little effect on blood pressure, it does include salt, which is known to raise blood pressure when eaten in large quantities. And, after the chi-square test, the findings were shown to be statistically significant for both 1 teaspoon and 112 teaspoons of coffee. Ground/black coffee is coffee that has been brewed without the addition of a creamer combination; however, in this research, there is also brewed/black coffee that has been prepared with the addition of a creamer mixture and is still classified as brewed/black coffee.

Coffee is rich in soluble fiber and has been linked to polyphenols, which are antioxidants (antioxidants). It is well known that coffee has a significant amount of potassium. Polyphenols are anti-atherosclerotic and have been shown to enhance vascular function. Sodium and water are excreted more readily by the body when potassium is present. Potassium lowers both systolic and diastolic blood pressure by blocking the release of renin. This results in a reduction in plasma volume, cardiac output, and peripheral pressure, all of which contribute to a decrease in blood pressure. Caffeine's stimulant effects may be countered by polyphenols and potassium.

Copyright $@$ ( 2021, International Journal Papier Advance and Scientific Review, Under the license CC BY-SA 4.0

DOI: https://doi.org/10.47667/ijpasr.v2i2.111 
When consumed in a specific amount, coffee may help to decrease blood pressure. This is due to the high potassium level found in coffee. Caffeine has an antagonistic action on adenosine receptors that is competitive in nature. Adenosine is a neuromodulator that has the ability to modulate a wide range of activities in the central nervous system. This has an effect on vasoconstriction and raises total peripheral resistance, which will cause blood pressure to rise as a result of the increased resistance.

\section{The Effect of the Amount of Coffee on Increasing Blood Pressure}

This research's findings on the impact of coffee intake on raising blood pressure were based on the results of bivariate analysis, which revealed that there is a relationship between the quantity of coffee consumed and increasing blood pressure in this study.

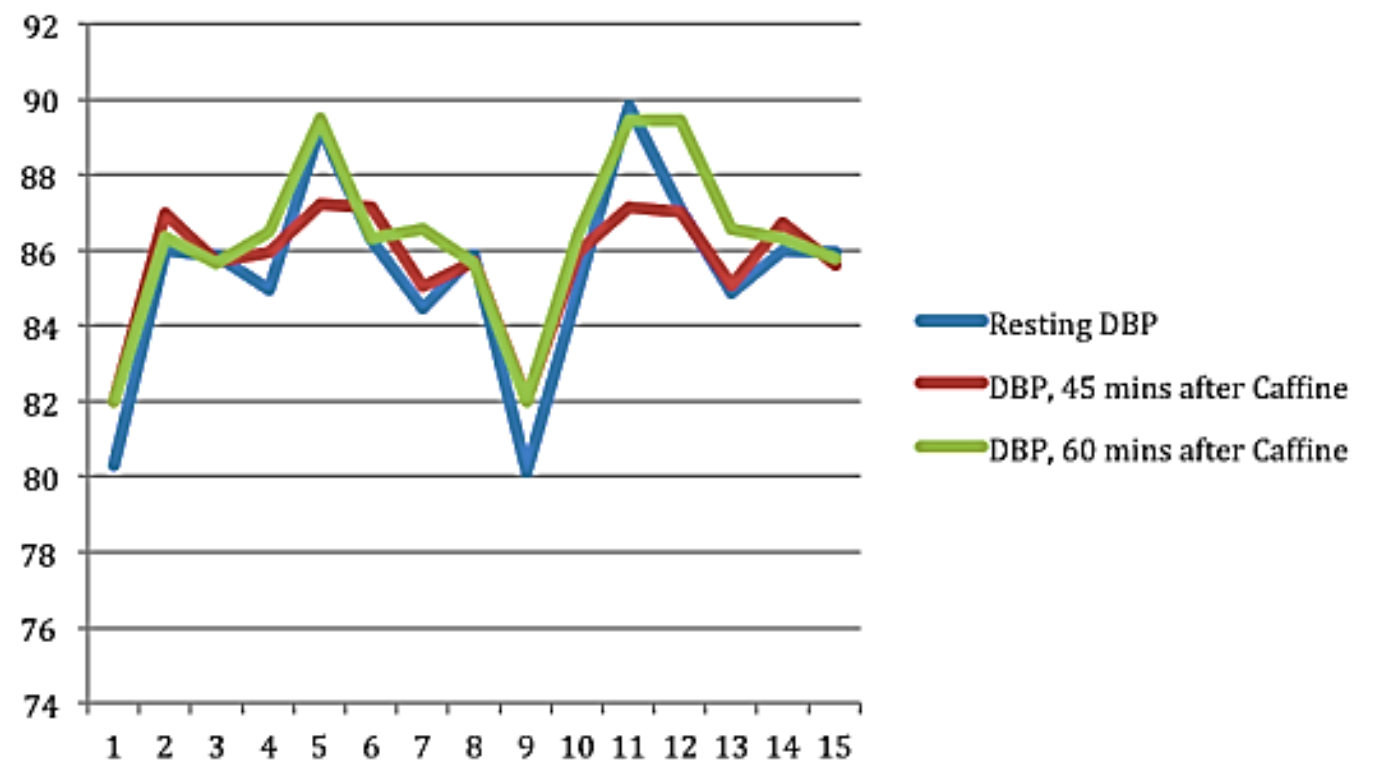

Figure 1. Effect of Caffeine on DBP

As a result of the caffeine in coffee, the central nervous system will be stimulated, resulting in an increase in blood pressure and muscular activity, as well as stimulation of the liver, which will release sugar molecules into the circulation, resulting in an increase in energy generation. Given the fact that caffeine possesses endogenous adenosine antagonist characteristics, it has the potential to induce vasoconstriction and raise peripheral vascular resistance in certain individuals. Within 30 minutes to two hours, this device is completely operational and ready to use. Several studies have shown that consuming coffee in quantities more than 300 grams per day (in the size of a cup) significantly increases the risk of hypertension by 4.12 times compared to individuals who do not consume coffee in this amount.

\section{Effect of Frequency of Drinking Coffee on Increased Blood Pressure}

According to the findings of this research, the impact of coffee intake on raising blood pressure can be inferred to be a result of the relationship between the frequency of coffee consumption and increasing blood pressure. We believe this is related to the body's tolerance to caffeine or coffee intake over a period of prolonged use.

After 18 hours, the sugar and creamer in instant coffee activate the adrenal glands, which may lead to a rise in the level of one of the stressors. It is generally believed that caffeine in coffee has the ability to raise blood pressure and heart rate, and that this is the root cause of the 
majority of chronic stress experienced throughout the workday. Most of the time, this impact will last until the evening, just before sleep. We feel restless and restless after drinking coffee, as a result of this. Coffee also includes an ingredient known as a terpenoid, which has been shown to raise cholesterol levels in the bloodstream. In certain cases, this may result in the arteries being clogged and as a consequence, the blood vessels working too hard.

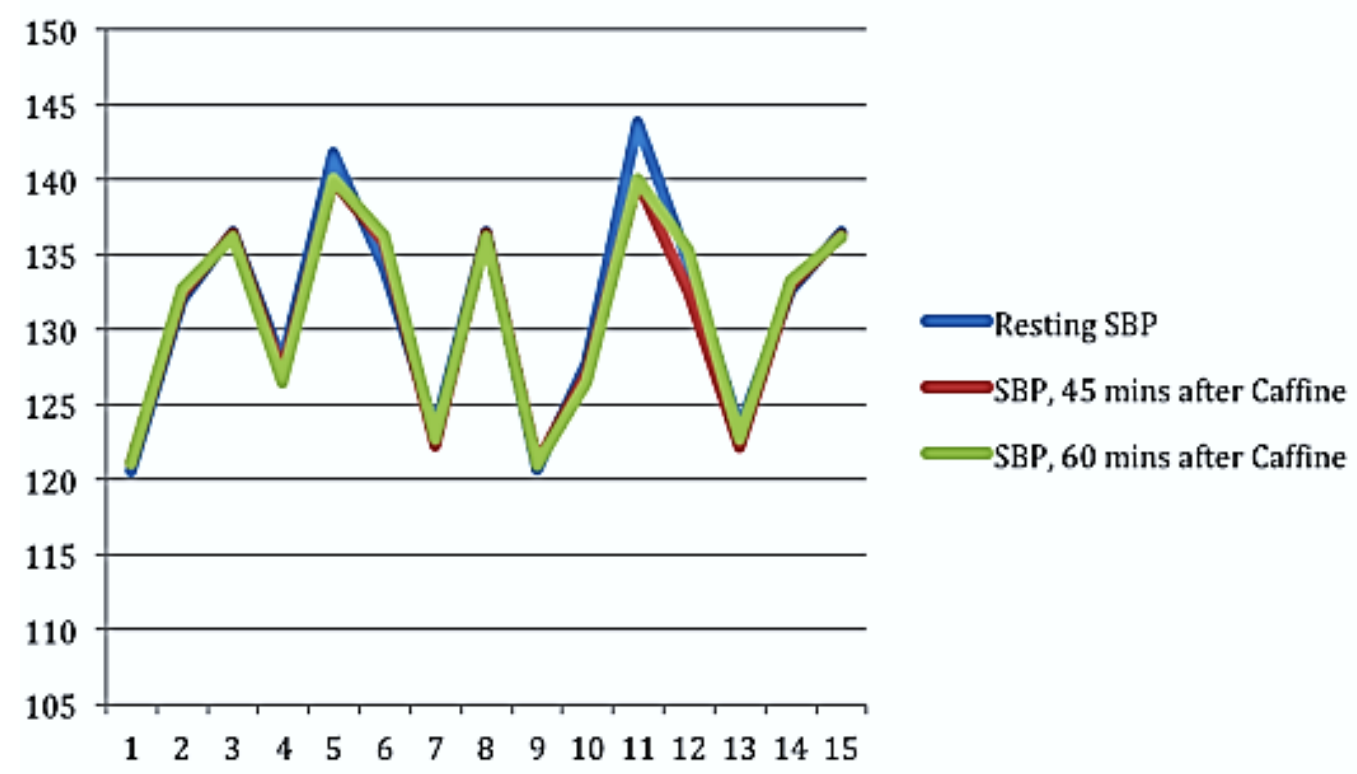

Figure 2. Effect of Caffeine on SBP

This study has many limitations, including a restricted amount of time, a limited number of research participants, and a limited variety of research topics, resulting in only observations of impacts being carried out in a short period of time. There is a restriction to the number of participants, with just one experimental group being used. As a result, further investigation is required. One of the research's limitations is that it does not take into account genetic variables in the participants. Inherent genetic variables influence the body's susceptibility to reacting to coffee, which has an effect on blood pressure in certain people. Individuals with a genetically slow caffeine metabolism may be at increased risk for developing heart disease if they consume large amounts of caffeine.

\section{Conclusion}

The relationship between coffee consumption and a rise in blood pressure demonstrated that the findings were linked and significant. When it comes to raising blood pressure, the amount of coffee consumption has been shown to have a statistically significant connection with the condition. The impact of the quantity of coffee consumed on the rise in blood pressure yielded findings that were linked to and significant. The impact of coffee consumption frequency on blood pressure elevation was shown to be statistically significant or related.

\section{References}

Buscemi, S., Verga, S., Batsis, J. A., Donatelli, M., Tranchina, M. R., Belmonte, S., ... \& Cerasola, G. (2010). Acute effects of coffee on endothelial function in healthy subjects. European journal of clinical nutrition, 64(5), 483-489.

Choi, E. H., Blasiak, A., Lee, J., \& Yang, I. H. (2019). Modulation of neural activity for myelination in the central nervous system. Frontiers in neuroscience, 13, 952. 
Choudhury, H., Chellappan, D. K., Sengupta, P., Pandey, M., \& Gorain, B. (2019). Adenosine receptors in modulation of central nervous system disorders. Current pharmaceutical design, 25(26), 2808-2827.

Goldstein, E. R., Ziegenfuss, T., Kalman, D., Kreider, R., Campbell, B., Wilborn, C., ... \& Antonio, J. (2010). International society of sports nutrition position stand: caffeine and performance. Journal of the International Society of Sports Nutrition, 7(1), 1-15.

Grgic, J., Sabol, F., Venier, S., Mikulic, I., Bratkovic, N., Schoenfeld, B. J., ... \& Mikulic, P. (2019). What dose of caffeine to use: acute effects of 3 doses of caffeine on muscle endurance and strength. International journal of sports physiology and performance, $15(4), 470-477$.

Grgic, J., Trexler, E. T., Lazinica, B., \& Pedisic, Z. (2018). Effects of caffeine intake on muscle strength and power: a systematic review and meta-analysis. Journal of the International Society of Sports Nutrition, 15(1), 1-10.

Kaparianos, A., \& Argyropoulou, E. (2011). Local renin-angiotensin II systems, angiotensinconverting enzyme and its homologue ACE2: their potential role in the pathogenesis of chronic obstructive pulmonary diseases, pulmonary hypertension and acute respiratory distress syndrome. Current medicinal chemistry, 18(23), 3506-3515.

Mussatto, S. I., Machado, E. M., Martins, S., \& Teixeira, J. A. (2011). Production, composition, and application of coffee and its industrial residues. Food and Bioprocess Technology, 4(5), 661-672.

Omondi, I. (2011). Changing the political landscape of a nation (Vol. 9). Mvule Africa Publishers.

Papamichael, C. M., Aznaouridis, K. A., Karatzis, E. N., Karatzi, K. N., Stamatelopoulos, K. S., Vamvakou, G., ... \& Mavrikakis, M. E. (2005). Effect of coffee on endothelial function in healthy subjects: the role of caffeine. Clinical Science, 109(1), 55-60.

Wei, C. J., Li, W., \& Chen, J. F. (2011). Normal and abnormal functions of adenosine receptors in the central nervous system revealed by genetic knockout studies. Biochimica et biophysica acta (BBA)-biomembranes, 1808(5), 1358-1379.

Weinberg, B. A., \& Bealer, B. K. (2004). The world of caffeine: the science and culture of the world's most popular drug. Routledge. 